\title{
Neutralizing antibodies derived from the B cells of 1918 influenza pandemic survivors
}

\author{
Xiaocong Yu ${ }^{1}$, Tshidi Tsibane ${ }^{2}$, Patricia A. McGraw ${ }^{1}$, Frances S. House ${ }^{1}$, Christopher J. \\ Keefer $^{1}$, Mark D. Hicar ${ }^{1}$, Terrence M. Tumpey ${ }^{3}$, Claudia Pappas ${ }^{2,3}$, Lucy A. Perrone ${ }^{3}$, \\ Osvaldo Martinez ${ }^{2}$, James Stevens ${ }^{3,4}$, Ian A. Wilson ${ }^{4}$, Patricia V. Aguilar ${ }^{2}$, Eric L. \\ Altschuler ${ }^{5}$, Christopher F. Basler ${ }^{2}$, and James E. Crowe Jr. ${ }^{1}$ \\ ${ }^{1}$ Departments of Pediatrics and of Microbiology and Immunology, Vanderbilt University Medical \\ Center, Nashville, TN 37232, USA \\ ${ }^{2}$ Department of Microbiology, Mount Sinai School of Medicine, New York, NY 10029, USA \\ ${ }^{3}$ Influenza Division, Centers for Disease Control and Prevention, Atlanta, GA 30333, USA \\ ${ }^{4}$ Department of Molecular Biology and Skaggs Institute for Chemical Biology, The Scripps Research \\ Institute, La Jolla, CA 92037, USA \\ ${ }^{5}$ Department of Physical Medicine and Rehabilitation, University of Medicine \& Dentistry of New \\ Jersey, Newark, NJ 07103, USA
}

\begin{abstract}
Investigation of the human antibody response to influenza virus infection has been largely limited to serology, with relatively little analysis at the molecular level. The 1918 H1N1 influenza virus pandemic was the most severe of the modern era ${ }^{1}$. Recent work has recovered the gene sequences of this unusual strain ${ }^{2}$, so that the 1918 pandemic virus could be reconstituted to display its unique virulence phenotypes ${ }^{3,4}$. However, little is known about adaptive immunity to this virus. We took advantage of the 1918 virus sequencing and the resultant production of recombinant 1918 hemagglutinin (HA) protein antigen to characterize at the clonal level neutralizing antibodies induced by natural exposure of survivors to the 1918 pandemic virus. In our study, each of 32 individuals tested that were born in or before 1915 exhibited seroreactivity with 1918 virus, nearly 90 years after the pandemic. Seven of 8 donor samples tested had circulating B cells that secreted antibodies that bound $1918 \mathrm{HA}$. We isolated B cells from subjects and generated five monoclonal antibodies that exhibited potent neutralizing activity against 1918 virus from three separate donors. These antibodies also cross-reacted with the genetically similar HA of a 1930 swine H1N1 influenza strain, but not with HAs of more contemporary human influenza viruses. The antibody genes exhibited an unusually
\end{abstract}

Correspondence should be addressed to JEC (James.Crowe@ vanderbilt.edu), CFB (Chris.Basler@mssm.edu) or ELA (Eric.Altschuler@umdnj.edu).

Supplementary Information is linked to the online version of the paper at www.nature.com/nature.

Author Contributions XY and TT contributed equally to this work. XY, PAM, MDH and FSH made and cloned the mAbs, sequenced antibody genes, and performed IF experiments, CJK performed biosensor studies, TMT, CP, and LAP designed and performed in vivo studies, OM sequenced the HA genes of the H1N1 viruses used in this study and performed ELISA assays with these viruses. PVA assisted with HAI and neutralization assays and with cloning of recombinant HA molecules. JS and IAW provided recombinant HA; ELA led the clinical recruitment, ELA, CFB and JEC conceived of the experimental plan. CFB and JEC wrote the manuscript. All authors discussed the results and commented on the manuscript.

Antibody nucleotide sequences are deposited in GenBank, accession numbers EU169674 through EU169679 and EU825947 through EU825950.

Reprints and permissions information is available at npg.nature.com/reprints and permissions.

The authors declare no competing financial interests. 
high degree of somatic mutation. The antibodies bound to the 1918 HA protein with high affinity, exhibited exceptional virus neutralizing potency, and protected mice from lethal infection. Isolation of viruses that escaped inhibition suggested that the antibodies recognize classical antigenic sites on the HA surface. Thus, these studies reveal that survivors of the 1918 influenza pandemic possess highly functional, virus-neutralizing antibodies to this uniquely virulent virus, and that humans can sustain circulating B memory cells to viruses for many decades after exposure - well into the tenth decade of life.

Recent studies suggest the $1918 \mathrm{H} 1 \mathrm{~N} 1$ influenza virus was of avian origin ${ }^{2,5}$, and is capable of inducing strong systemic cytokine responses that likely contribute to pathogenesis ${ }^{4,6}$. Little is known about naturally occurring adaptive immunity to this virus; however, some elderly survivors are still living. We sought to determine whether survivors exhibited evidence of acquired immunity to the virus. Expression of the 1918 HA antigen allowed us to identify and characterize protective antibodies induced by natural exposure of humans to the 1918 pandemic virus.

We identified a panel of 32 subjects aged 91-101 years (i.e., aged 2 to 12 years in 1918), many of whom recalled a sick family member in the household during the pandemic, which suggested direct exposure to the virus. Of the subjects tested, $100 \%$ exhibited serum neutralizing activity against the 1918 virus (mean titer 1:562), and 94\% had serologic reactivity to the 1918 HA (as indicated by hemagglutination inhibition assay (HAI) titers of 1:40 or greater; mean titer 1:396), even though these samples were obtained nearly 90 years after the pandemic. In contrast, subjects born after the pandemic exhibited markedly lower rates of positive serum neutralizing tests against 1918 virus ( 9 of 10 subjects born 1926-35 had titers $<1: 100,9$ of 10 subjects born 1936-45 had titers $\leq 1: 40,9$ of 10 subjects born 1946-55 had titers $\leq 1: 40$ ). Individual serologic results are shown in Supplementary Table 1.

Peripheral blood mononuclear cells from eight subjects were isolated and B lymphoblastic cell lines generated by transformation; blood from almost all donors tested (7 of 8) yielded transformed cells secreting antibodies binding 1918 HA protein. Supernates from 30 wells of a total of 6578 wells tested contained 1918 HA-specific antibodies, suggesting a minimal frequency of circulating 1918 HA-specific B cells in the donors of approximately 1 in $4.6 \times$ $10^{6}$. We collected transformed cells from the wells corresponding to supernates exhibiting the highest levels of specific binding to the 1918 HA (derived from five donors) and fused them to the HMMA2.5 nonsecreting myeloma partner ${ }^{7}$ using an electrofusion technique ${ }^{8}$. We isolated 17 unique hybridoma cell lines that secreted antibodies reactive with the $1918 \mathrm{HA}$ from cell lines derived from four of five donors and then segregated the lines by limiting dilution to yield monoclonal antibody (mAb) secreting clones. Our screening identified five independent lines with HAI activity against 1918 from three separate donors, which we biologically cloned, and designated mAbs 1I20, 1F1, and 2B12 (donor 6), mAb 4D20 (donor 4) and mAb 2D1 (donor 23).

Sequence analysis of the antibody genes from the clones revealed that the five mAbs were distinct and very highly mutated. Genetic features of the mAbs are shown in Table 1. It was of interest that the $1 \mathrm{~F} 1,2 \mathrm{~B} 12$ and $2 \mathrm{D} 11$ clones shared use of the $\mathrm{V}_{\mathrm{L}} 1-44 * 01$ gene segment, suggesting a particular fitness for binding of the 1918 virus HA by the CDR1/2 light chain loops encoded by this $\mathrm{V}_{\mathrm{L}}$ gene segment. The three clones, however, were clearly independent as they differed in the location of somatic mutations, $\mathrm{J}_{\mathrm{L}}$ segment $(1 \mathrm{~F} 1)$ and in heavy chain pairing. The numbers of somatic mutations in the variable regions were exceptionally large, almost twice the median number of 18 mutations found in class-switched memory cells in randomly selected human $\mathrm{B}$ cells ${ }^{9}$. These data likely suggest recurrent optimization of binding affinity through multiple rounds of somatic hypermutation and selection in vivo. 
Purified mAbs were assessed by ELISA against a series of representative $20^{\text {th }}$ century H1N1 viruses including human isolates from 1918, 1943, 1947, 1977 and 1999. We also examined reactivity with influenza A/Swine/Iowa/15/30 (H1N1) virus, as the HA sequence of this virus more closely resembles the 1918 HA sequence than does the sequence of any other existing isolate. The mAbs bound to the 1918 HA, with clear cross-reactivity with the 1930 strain, suggesting the remote origin of the antibodies (Figure 1 and Supplementary Figure 1). The 1F1 clone also bound to a minimal degree to the 1977 strain, a virus that is almost identical to isolates from the early $1950 \mathrm{~s}^{10}$, and minimally to a 1943 isolate, but not to other post-1930 strains. The mAbs also bound to the corresponding HA expressed on the surface of mammalian cells following transfection of a cDNA encoding the $1918 \mathrm{HA}$ as detected by immunofluorescence microscopy (Supplementary Figure 2). The mAbs did not bind to influenza H3, B, or H5 HA proteins in ELISA (data not shown). All five antibodies proved to have very high affinities for recombinant 1918 HA protein when tested by surface plasmon resonance, ranging from $5.4 \times 10^{-9}$ to $4.8 \times 10^{-11} \mathrm{M}$ (Table 1).

We tested five purified mAbs from three separate donors for inhibitory activity in an HAI assay using 1918 virus-like particles (VLPs) or a panel of H1N1 viruses. The mAbs exhibited specific binding for "old" viruses, including the 1918 virus or viruses that were genetically similar to the 1918 virus (Table 2 and Supplementary Table 2). Specifically, all five mAbs reacted with 1918 HA by ELISA, by HAI with VLPs of the 1918 strain or with the highly genetically similar influenza A/Swine/30 (H1N1) virus (Sw/30), and by neutralizing assay with reconstituted 1918 virus. Comparable HAI activities were obtained for both 1918 and Sw/30 VLPs in these assays (Table 2). The five clones exhibited specific neutralizing activities ranging from 0.32 to 0.97 $\mu \mathrm{g} / \mathrm{mL}$ and HAI activities of 0.18 to 0.47 against the 1918 virus. In contrast, the same mAbs failed to interact with or inhibit human H1N1 viruses isolated in 1943, 1947 or 1999 (Supplementary Table 2). The 1F1 antibody bound and neutralized the 1977 virus, albeit to a lesser degree than either 1918 or Sw/30 viruses (exhibiting a specific HAI activity of $0.16 \mu \mathrm{g} /$ $\mathrm{mL}$ against the 1977 virus), and to a minimal degree the 1943 virus (Supplementary Table 2). Again, only 1F1 displayed neutralizing activity against the 1943 or 1977 viruses, with a specific activity of 1.8 and $0.88 \mu \mathrm{g} / \mathrm{mL}$ respectively.

We selected antibody escape mutants for three mAbs using the $\mathrm{Sw} / 30$ virus. Nucleotide sequence analysis of the HA genes from these viruses revealed that they had acquired mutations in classical antigenic regions of HA. The 2B12 mutants possessed mutations at residue 166 (K166Q, K166E or K166P) which lies within the Sa antigenic site (Supplementary Figure 3), using the previous numbering scheme ${ }^{11}$. Escape mutants for $1 \mathrm{~F} 1$ and $1 \mathrm{I} 20$ possessed an identical mutation, $\mathrm{P} 186 \mathrm{H}$, a residue adjacent to the previously defined $\mathrm{Sb}$ antigenic site (Supplementary Figure 3), which encompasses the $\alpha$-helix of the receptor binding site in the 1918 HA structure ${ }^{11-13}$. As expected, incorporation of the Sb antigenic site mutation into VLPs reduced (1I20) or eliminated (1F1) activity of the corresponding mAbs in an HAI assay without affecting binding of 2B12 (Table 2). The 2D1 and 4D20 mAbs also exhibited reduced HAI activity against the site $\mathrm{Sb}$ mutant viruses and VLPs, suggesting they also bound to this site. In contrast, incorporation of the Sa mutation into VLPs abolished activity of 2B12 in the HAI assay, but did not affect the activity of the other four mAbs (Table 2). The 1918 and Sw/30 virus HAs differ by only a single amino acid in each of the Sa and Sb sites (Supplementary Figure 3), explaining why these mAbs cross-neutralize both viruses. In contrast, the 1943 isolate contains 7 changes in the Sa site and 7 changes in the $\mathrm{Sb}$ site, relative to the $1918 \mathrm{HA}$ sequence, explaining the partial or complete loss of neutralizing ability of the mAbs for these later isolates. Strikingly, 9 of the 12 residues at the Sb site differed between the 1918 and the 1977 virus. The ability of $1 F 1$ to cross-neutralize the 1977 virus is worthy of further investigation, since these data raise the possibility that $1 \mathrm{~F} 1$ recognizes a novel, more broadly neutralizing epitope. Alternatively, the data may represent epitope recycling such that, despite significant sequence divergence, the $1 \mathrm{~F} 1$ epitope reappeared. In either case, further 
characterization of this mAb may suggest strategies to elicit enhanced cross-protective immunity to influenza A viruses of a particular HA subtype.

We tested the five mAbs for therapeutic efficacy in an established mouse model of infection. Mice were inoculated by the intranasal route with the previously reconstructed 1918 virus, and morbidity (measured by weight loss), mortality, and virus replication, were assessed as previously described ${ }^{3}$. Each of the five 1918-specific mAbs tested exhibited therapeutic efficacy when administered one day following infection, preventing death of animals (Table 2). Mice treated with a control (H5 HA-specific) human mAb or human IgG did not survive. Reduced weight loss and lower levels of virus replication in lungs of anti-1918 antibody treated mice on day 4 after infection also revealed a significant protective effect that correlated well with survival data. At lower doses, the mAbs caused a statistically-significant delay to death, relative to the controls.

These studies suggest that B cells responding to viral infections, or their progeny, survive for the life of the host, even nine or more decades after exposure. It is well-established that a subset of plasma cells is long-lived ${ }^{14}$, and these cells contribute to durable humoral immune responses ${ }^{15}$, such as that observed after childhood smallpox vaccination ${ }^{16-19}$. Memory B cell pools also can be long-lived, sustained in part by antigen-independent polyclonal stimuli ${ }^{20}$. It is difficult to be absolutely certain that the mAbs isolated here were first stimulated by exposure during the 1918 pandemic. However, the clinical history of the subjects and the high functional specificity of the mAbs for the 1918 strain strongly suggest that recent exposures do not account for this immunity. Likely, boosting by antigenically-related viruses in the early decades of the $20^{\text {th }}$ century may have contributed to the ability of these subjects to sustain these B cells. The variable genes of five independent human neutralizing mAbs exhibited a very high frequency of somatic mutations, associated with strong binding constants and high potency. The in vivo efficacy of treatment with these $\mathrm{mAb}$ shows that the development of functional adaptive immunity to the pandemic virus did occur in survivors of the 1918 pandemic. It has long been known that infusion of neutralizing antibodies can protect mice from lethal influenza virus infection, and transfusion of convalescent blood products to 1918 influenza victims may have had a beneficial effect ${ }^{21}$. Thus, the mAbs described here could serve as potential therapeutics for a re-emergent 1918-like virus. The techniques described herein suggest that it may be possible to recover human antibodies that display a wide array of specificities corresponding to the viruses and other pathogens that have infected an individual during their lifetime.

\section{Methods Summary}

Recombinant 1918 virus HA (A/South Carolina/1/1918) was produced as described ${ }^{11}$. PBMCs were obtained from volunteers born in 1915 or earlier. Hybridomas were generated from EBVtransformed B cell lines by electrofusion to the HMMA2.5 cell line ${ }^{7,8}$. When hybridoma lines formed colonies in the presence of selecting drugs, lines were cloned by limiting dilution. Secreted mAbs were concentrated and purified by FPLC. The isotype and subclass of secreted $\mathrm{mAbs}$ were determined by ELISA. Nucleotide sequences of variable gene segments were determined by automated sequence analysis of cloned cDNA ${ }^{22}$. Identity of the gene segments and mutations from the germline sequences were determined by alignment using the ImMunoGeneTics database ${ }^{23}$. Viruses were propagated in 10-day-old embryonated chicken eggs. Influenza A/South Carolina/1/18 (H1N1) virus was prepared as previously described ${ }^{3}$.

Expression plasmids encoding the 1918 HA and NA proteins were described ${ }^{24,25}$. Binding of antibodies was determined using 1918 virus-like particles (VLPs) or influenza A viruses as the coating antigen in ELISA. VLPs were produced by co-transfection of 293T cells with expression plasmids for the $1918 \mathrm{HA}$ and $1918 \mathrm{NA}$, consistent with a recent report ${ }^{26}$. HAI assays of sera or mAbs were performed according to standard protocols using chicken red blood cells ${ }^{27}$. For microneutralization assay, 10 TCID $_{50}$ units of virus was preincubated with 
dilutions of sera or mAb and then used to infect Madin-Darby canine kidney cells in 96-well plates, as described ${ }^{28,29}$. The kinetic interaction of mAbs with 1918 HA protein was determined by surface plasmon resonance. Antibody escape mutants were isolated by treatment of Sw/30 virus with excess antibody as described ${ }^{12,} 30$. Mice were inoculated intranasally with $5 \mathrm{LD}_{50}$ of the 1918 virus. At $24 \mathrm{hrs}$ after inoculation, we administered 1918-specific mAb or control antibodies to each mouse. Mice were observed for weight loss or death. Subsets of animals were sacrificed for virus titer.

\section{Methods}

\section{Subjects}

Volunteers born in 1915 or earlier were recruited at the UMDNJ site. We obtained from each subject $50 \mathrm{~mL}$ of peripheral venous blood following written informed consent.

\section{Antigen}

Recombinant A/South Carolina/1/1918 virus HA was generated in a baculovirus expression system and purified, as described ${ }^{11}$.

\section{Production of mAbs}

PBMCs were obtained from eight donors by density gradient centrifugation of whole heparinized blood. B cells were transformed in 384-well plates with approximately $1000 \mathrm{~B}$ cells per well by in vitro culture in medium with CpG ODN 10103 (Coley) and EBV (supernate from cell line B95.8). Supernates from the resulting cell lines were tested for reactivity with 1918 HA by ELISA. Hybridomas were generated using cells from wells containing reactive lines by fusion to the HMMA2.5 nonsecreting myeloma cell line ${ }^{7}$ using electrofusion followed by HAT/ouabain drug selection, as described ${ }^{8}$. When hybridomas formed colonies, lines were screened using anti-1918 HA ELISA; positive lines were cloned by limiting dilution.

Supernates of high-density cultures were produced in CELLine Devices (BD Biosciences). Purified and concentrated preparations of each of the antibodies were prepared by FPLC using protein $\mathrm{G}$ conjugated resin on an AKTA instrument (GE Healthcare) followed by concentration and buffer exchange using ultra centrifugal filter devices (Millipore).

\section{Characterization of mAbs}

The isotype and subclass of secreted mAbs were determined by ELISA. Nucleotide sequences of variable gene segments were determined from cloned cDNA generated by RT-PCR amplification of cellular mRNA using variable gene specific primers designed to amplify antibody genes from all gene families ${ }^{22}$. Identity of the gene segments and mutations from the germline sequences were determined by alignment using the ImMunoGeneTics database (http://imgt.cines.fr:8104) ${ }^{23}$.

\section{Viruses and plasmids}

The following viruses were propagated in 10-day-old embryonated chicken eggs: A/Weiss/43; A/FM/1/47; A/USSR/92/77; A/New Caledonia/20/99; A/swine/Iowa/15/30. The sequences of the HA genes of these viruses used were confirmed by RT-PCR and cDNA sequence analysis. Influenza A/South Carolina/1/18 virus was prepared as described ${ }^{3}$. Expression plasmids encoding the $1918 \mathrm{HA}$ and NA proteins were described previously $24,25$.

\section{ELISA}

Equivalent HA units of 1918 virus-like particles (VLPs) or of influenza A viruses were diluted in coating buffer (51-2713KC; BD) and adsorbed overnight onto ELISA plates (Nunc). Plates were washed with PBS/0.05\% Tween-20 and blocked using blocking solution (555213; BD) 
or PBS containing 5\% FCS at RT for 1 hour. Diluted mAbs were added to plates and incubated at $4{ }^{\circ} \mathrm{C}$ for 16 hours. Plates were washed and incubated with HRP-conjugated goat anti-human $\operatorname{IgG}$ (H10507; Caltag) for 1 hour. Washed plates were developed using TMB substrate, and the reaction was stopped using $2 \mathrm{~N}$ sulfuric acid. Optical density was read at $450 \mathrm{~nm}$ using an ELISA plate reader.

\section{Production of VLPs}

VLPs were produced by co-transfection of $10^{6} 293 \mathrm{~T}$ cells with $1 \mu \mathrm{g}$ each of expression plasmids for the $1918 \mathrm{HA}$ and NA. Two days post-transfection, supernatants were harvested and assayed for HA activity. Our ability to produce these hemagglutinating particles in the absence of other viral proteins is consistent with a recent report ${ }^{26}$.

HAI assays of sera or mAbs were performed according to standard protocols ${ }^{27}$. Briefly, sera were diluted initially 1:10 in receptor-destroying enzyme from Vibrio cholerae (Denka Seiken, Tokyo). Serial dilutions of sera or mAbs were pre-incubated with $8 \mathrm{HA}$ units of virus per well. Chicken RBCs were added to a final concentration of $0.5 \%$, and the plate was incubated on ice for 30-60 minutes.

\section{Microneutralization assay}

10 TCID $_{50}$ units of virus was preincubated with dilutions of sera or mAb and used to infect MDCK cells in 96-well plates with six replicates, as described ${ }^{28}$. Neutralizing concentrations were defined as the reciprocal of the highest dilution of serum where $50 \%$ of wells were infected, as calculated by the method of Reed and Muench ${ }^{29}$. Specific neutralizing activity of $\mathrm{mAbs}$ was calculated as the lowest concentration of mAb that displayed activity.

\section{Biosensor studies}

The kinetic interaction of mAbs with recombinant 1918 HA protein was determined by surface plasmon resonance using a Biacore 2000 instrument (Biacore, Piscataway, NJ). Purified 1918 HA protein was diluted to $30 \mu \mathrm{g} / \mathrm{ml}$ in $10 \mathrm{mM}$ sodium acetate, $\mathrm{pH} 4.5$, and covalently immobilized at $5 \mu \mathrm{l} / \mathrm{min}$ by amine coupling to the dextran matrix of a CM5 sensor chip (Biacore $\mathrm{AB}$ ) with a target density of 1,200 response units (RU). Unreacted active ester groups were blocked with $1 \mathrm{M}$ ethanolamine. All five purified $1918 \mathrm{mAbs}$ and a human H5-specific influenza $\mathrm{mAb}$ (negative control) at different concentrations ranging from 5 to $500 \mathrm{nM}$ in HBS/ Tween-20 buffer (Biacore AB) were injected over the immobilized 1918 HA protein or reference cell surface. Association rates $\left(K_{o n}\right)$, dissociation rates $\left(K_{o f f}\right)$, and equilibrium dissociation constants $\left(K_{D}\right)$ were calculated by aligning the binding curves globally to fit a 2:1 Langmuir binding model using BIAevaluation 4.1 software. The goodness of each fit was based on the agreement between experimental data and the calculated fits, where the $X^{2}$ values were below 1.0.

\section{Selection and characterization of antibody escape mutants}

Mutants were isolated as described ${ }^{12,30}$. Briefly, escape mutant viruses were selected by treatment of $\mathrm{Sw} / 30$ virus with excess antibody, followed by recovery of neutralization resistant viruses in eggs. RNA was extracted from virus-infected allantoic fluid, then cDNA was generated by RT-PCR, cloned, sequenced, and aligned to previously determined $w t$ virus HA gene sequences.

\section{Animal studies}

Female BALB/c (8-week old) mice were inoculated intranasally with $5 \mathrm{LD}_{50}$ in a $50 \mu$ volume of the virulent reconstituted 1918 virus. At $24 \mathrm{hrs}$ after inoculation, we administered 200, 20, 2 or $0.2 \mu \mathrm{g}$ (approximately $10,1,0.1$ or $0.01 \mathrm{mg} / \mathrm{kg}$ ) of 1918 -specific mAb or a similarly 
prepared human mAb to H5 influenza HA (clone IE5), or an equal volume of human IgG, to each mouse, in groups of 11 (highest dose) or 5 (lower doses) mice. Mice were observed for 16 days for weight loss or death. Subsets of 3 animals treated with the highest dose were sacrificed on day 2 and 4 after infection and whole lungs were homogenized in $1 \mathrm{~mL}$ of sterile PBS. Virus titer in lung tissue homogenates was determined by plaque titration in MDCK cell monolayer cultures.

\section{Supplementary Material}

Refer to Web version on PubMed Central for supplementary material.

\section{Acknowledgments}

We thank Lisa Schoenherr, Keerti Sharma, Latasha Adams (supported by the UMDNJ Institute for the Elimination of Health Disparities), Carol Dokes, Camille Gaines, Bonnie Butter and Susan Rivera of the UMDNJ Clinical Research Center for assistance with subjects and Sandra Yoder and Bryan Briney of Vanderbilt for sample preparation. This work was supported by grants from the National Institutes of Health to JEC [U54 AI057157 (Southeast Regional Center of Excellence for Emerging Infections and Biodefense) and U19 AI057229], and CFB [U54 AI57158, (Northeast Biodefense Center), U19 AI62623 (Center for Investigating Viral Immunity and Antagonism (CIVIA) and P01 AI058113]. IAW and JS were supported in part by NIH (CA55896 and AI42266). PVA was supported by a fellowship awarded by the Northeast Biodefense Center (AI057158). We thank Peter Palese and Adolfo Garcia-Sastre, Mount Sinai School of Medicine, for useful advice and for providing H1N1 viruses, and Marshall Posner and Lisa Cavacini of Beth Israel Deaconess Medical Center for the HMMA2.5 cell line.

\section{References}

1. Johnson NP, Mueller J. Updating the accounts: global mortality of the 1918-1920 "Spanish" influenza pandemic. Bull Hist Med 2002;76:105-115. [PubMed: 11875246]

2. Taubenberger JK, et al. Characterization of the 1918 influenza virus polymerase genes. Nature 2005;437:889-893. [PubMed: 16208372]

3. Tumpey TM, et al. Characterization of the reconstructed 1918 Spanish influenza pandemic virus. Science 2005;310:77-80. [PubMed: 16210530]

4. Kobasa D, et al. Aberrant innate immune response in lethal infection of macaques with the 1918 influenza virus. Nature 2007;445:319-323. [PubMed: 17230189]

5. Taubenberger JK. The origin and virulence of the 1918 "Spanish" influenza virus. Proc Am Philos Soc 2006;150:86-112. [PubMed: 17526158]

6. Kash JC, et al. Genomic analysis of increased host immune and cell death responses induced by 1918 influenza virus. Nature 2006;443:578-581. [PubMed: 17006449]

7. Posner MR, Elboim H, Santos D. The construction and use of a human-mouse myeloma analogue suitable for the routine production of hybridomas secreting human monoclonal antibodies. Hybridoma 1987;6:611-625. [PubMed: 3436625]

8. Yu X, McGraw PA, House FS, Crowe JE Jr. An optimized electrofusion-based protocol for generating virus-specific human monoclonal antibodies. J Immunol Methods. In press. [PubMed: 18514220]

9. Tian C, et al. Evidence for preferential Ig gene usage and differential TdT and exonuclease activities in human naive and memory B cells. Mol Immunol 2007;44:2173-2183. [PubMed: 17196657]

10. Nakajima K, Desselberger U, Palese P. Recent human influenza A (H1N1) viruses are closely related genetically to strains isolated in 1950. Nature 1978;274:334-339. [PubMed: 672956]

11. Stevens J, et al. Structure of the uncleaved human H1 hemagglutinin from the extinct 1918 influenza virus. Science 2004;303:1866-1870. [PubMed: 14764887]

12. Caton AJ, Brownlee GG, Yewdell JW, Gerhard W. The antigenic structure of the influenza virus A/ PR/8/34 hemagglutinin (H1 subtype). Cell 1982;31:417-427. [PubMed: 6186384]

13. Brownlee GG, Fodor E. The predicted antigenicity of the haemagglutinin of the 1918 Spanish influenza pandemic suggests an avian origin. Phil Trans R Soc Lond B Biol Sci 2001;356:18711876. [PubMed: 11779386] 
14. Manz RA, Thiel A, Radbruch A. Lifetime of plasma cells in the bone marrow. Nature 1997;388:133134. [PubMed: 9217150]

15. Slifka MK, Antia R, Whitmire JK, Ahmed R. Humoral immunity due to long-lived plasma cells. Immunity 1998;8:363-372. [PubMed: 9529153]

16. Amanna IJ, Slifka MK, Crotty S. Immunity and immunological memory following smallpox vaccination. Immunol Rev 2006;211:320-337. [PubMed: 16824139]

17. Crotty $\mathrm{S}$, et al. Cutting edge: long-term B cell memory in humans after smallpox vaccination. $\mathbf{J}$ Immunol 2003;171:4969-4973. [PubMed: 14607890]

18. Hammarlund E, et al. Duration of antiviral immunity after smallpox vaccination. Nature Med 2003;9:1131-1137. [PubMed: 12925846]

19. Amanna IJ, Carlson NE, Slifka MK. Duration of humoral immunity to common viral and vaccine antigens. N Engl J Med 2007;357:1903-1915. [PubMed: 17989383]

20. Bernasconi NL, Traggiai E, Lanzavecchia A. Maintenance of serological memory by polyclonal activation of human memory B cells. Science 2002;298:2199-2202. [PubMed: 12481138]

21. Luke TC, Kilbane EM, Jackson JL, Hoffman SL. Meta-analysis: convalescent blood products for Spanish influenza pneumonia: a future H5N1 treatment? Ann Intern Med 2006;145:599-609. [PubMed: 16940336]

22. Weitkamp JH, et al. Generation of recombinant human monoclonal antibodies to rotavirus from single antigen-specific B cells selected with fluorescent virus-like particles. J Immunol Methods 2003;275:223-237. [PubMed: 12667686]

23. Ruiz M, et al. IMGT, the international ImMunoGeneTics database. Nucleic Acids Res 2000;28:219221. [PubMed: 10592230]

24. Tumpey TM, et al. Existing antivirals are effective against influenza viruses with genes from the 1918 pandemic virus. Proc Natl Acad Sci USA 2002;99:13849-13854. [PubMed: 12368467]

25. Glaser L, et al. A single amino acid substitution in 1918 influenza virus hemagglutinin changes receptor binding specificity. J Virol 2005;79:11533-11536. [PubMed: 16103207]

26. Chen BJ, Leser GP, Morita E, Lamb RA. Influenza virus hemagglutinin and neuraminidase, but not the matrix protein, are required for assembly and budding of plasmid-derived virus-like particles. $\mathrm{J}$ Virol 2007;81:7111-7123. [PubMed: 17475660]

27. Kendal, AP.; Skehel, JJ.; Pereira, MS., editors. World Health Organization Collaborating Centers for Reference and Research on Influenza. Concepts and Procedures for Laboratory-Based Influenza Surveillance. Centers for Disease Control and Prevention; Atlanta, GA: 1982. p. B17-B35.

28. Mozdzanowska K, et al. A pulmonary influenza virus infection in SCID mice can be cured by treatment with hemagglutinin-specific antibodies that display very low virus-neutralizing activity in vitro. J Virol 1997;71:4347-4355. [PubMed: 9151823]

29. Reed LJ, Muench H. A simple method of estimating fifty percent endpoints. Am J Hygiene 1938;27:493-497.

30. Yewdell JW, Webster RG, Gerhard WU. Antigenic variation in three distinct determinants of an influenza type A haemagglutinin molecule. Nature 1979;279:246-248. [PubMed: 86955] 

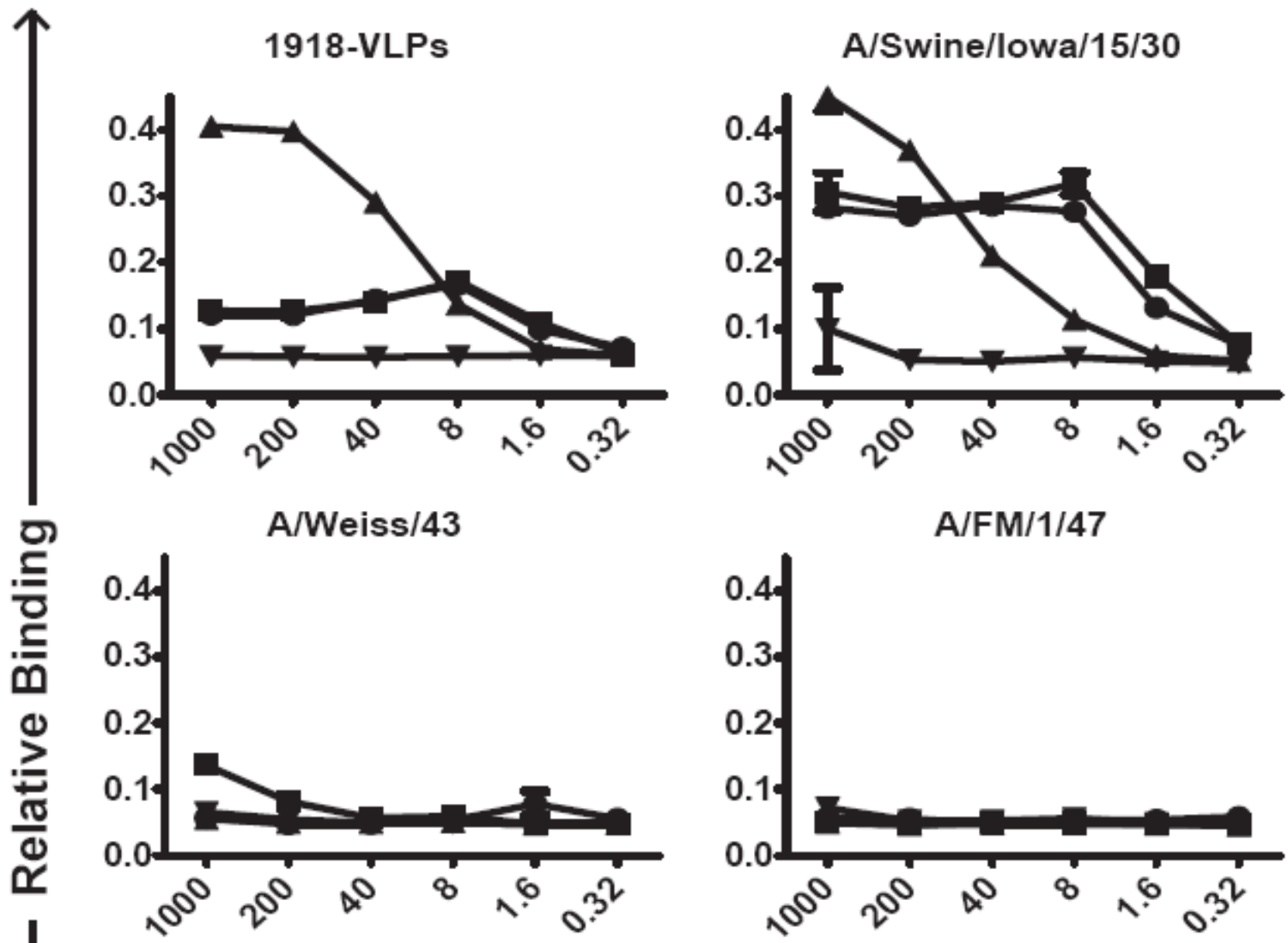

A/USSR/92/77

A/New Caledonia/20/99
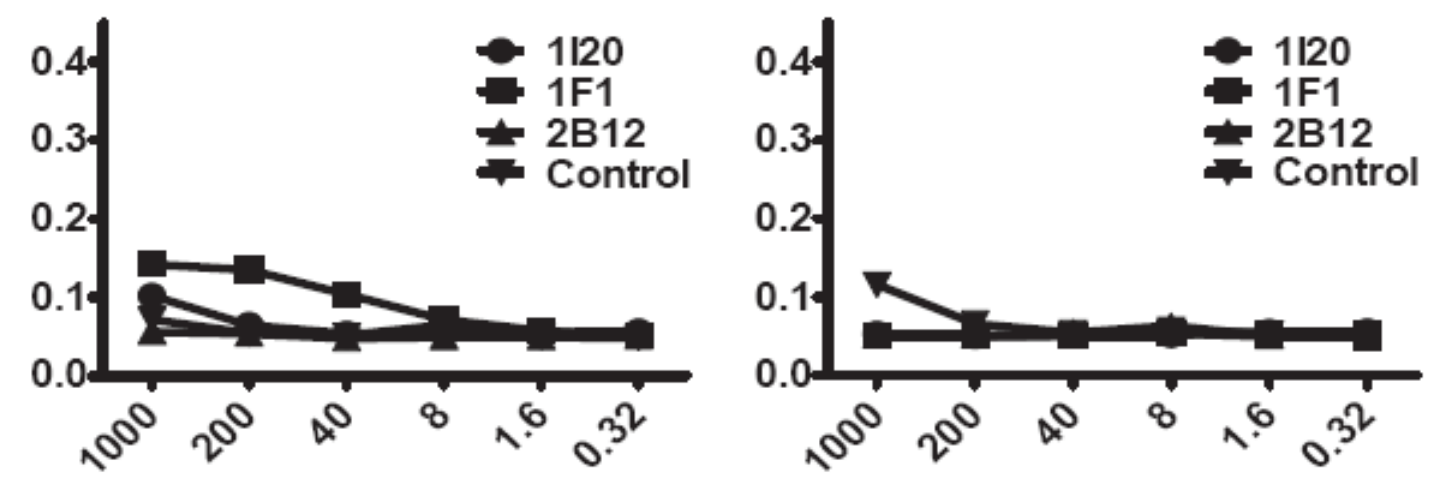

(ng/mL)

Figure 1. Binding of human mAbs to representative $20^{\text {th }}$ century H1N1 viruses

Equivalent HA units of 1918 virus-like particles, A/Iowa/Swine/30, A/Weiss/43, A/FM/1/47, A/USSR/92/77 and A/New Caledonia/20/99 influenza viruses were absorbed onto ELISA plates. An ELISA was performed using serial 1:5 dilutions of mAbs 1F1, 1I20, 2 B12 or an H5specific control mAb. Relative binding (y-axis) indicates optical density in ELISA binding assay to absorbed VLPs or virus. 


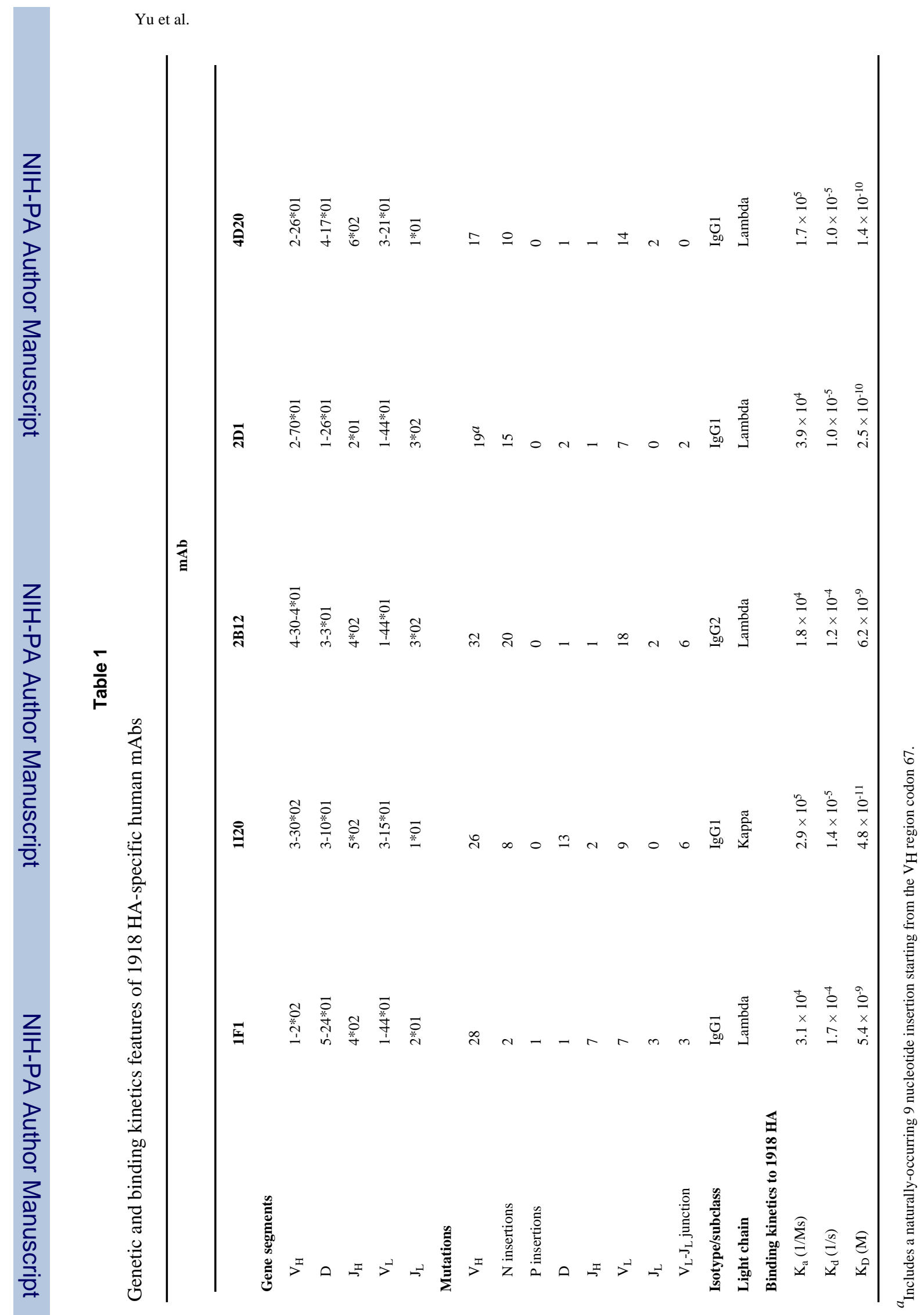

Page 10 


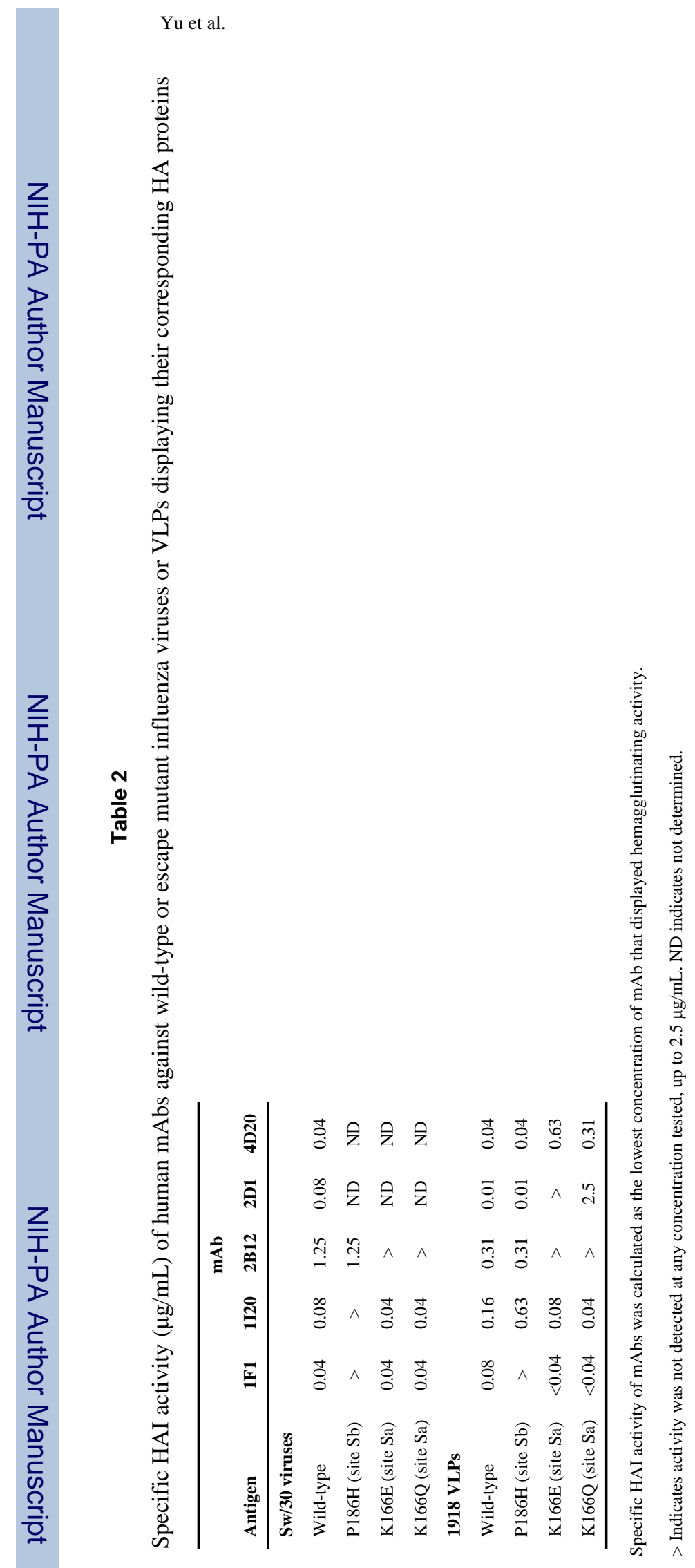

Nature. Author manuscript; available in PMC 2010 April 3. 


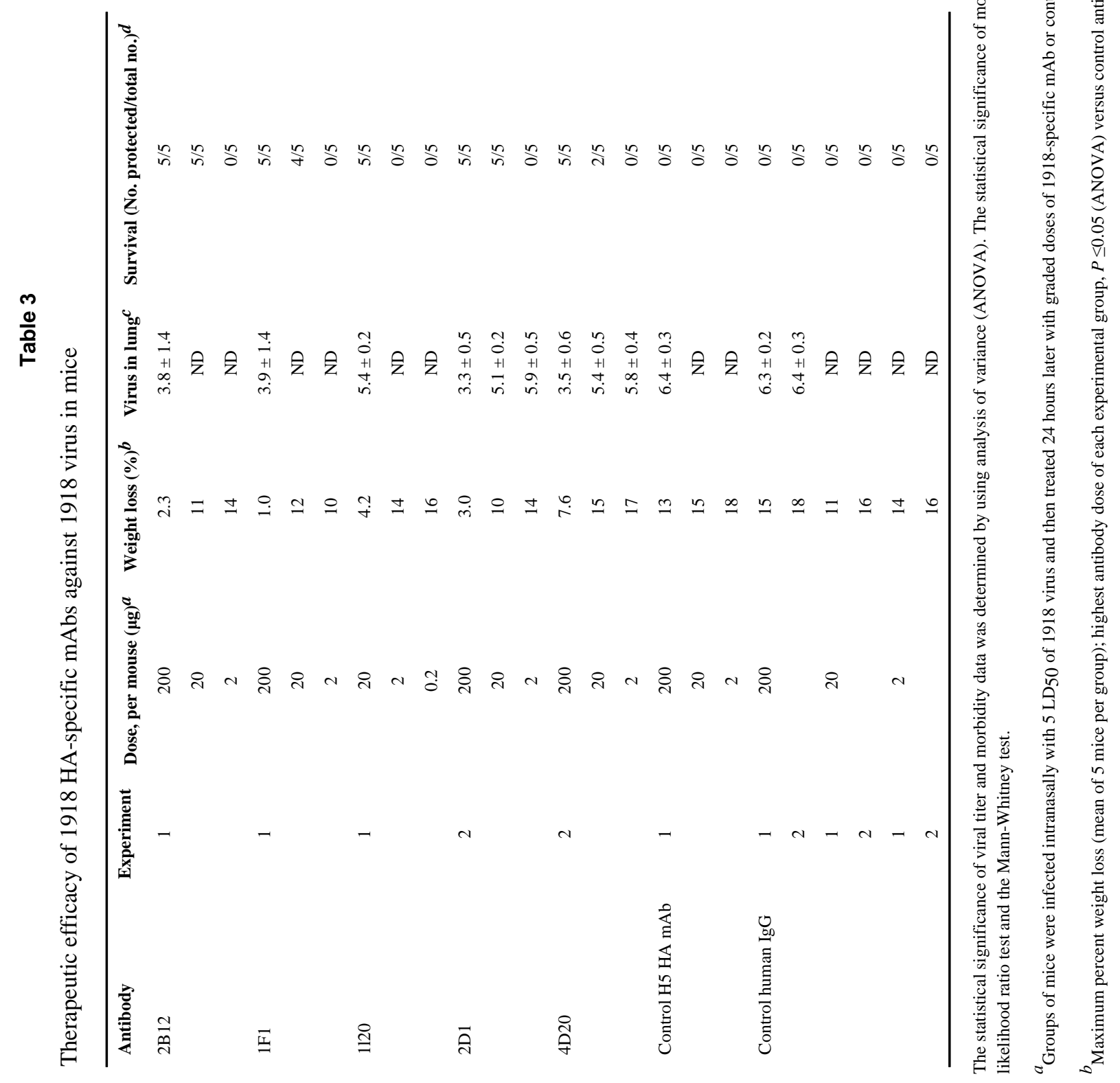


